\title{
X-Shooter Survey of Near-Infrared DIBs
}

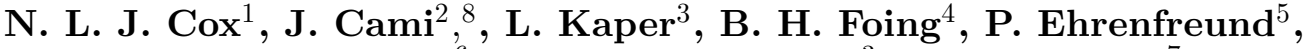 \\ B. B. Ochsendorf ${ }^{6}$, S. H. M. van Hooff ${ }^{3}$ and F. Salama \\ ${ }^{1}$ Instituut voor Sterrenkunde, KU Leuven, Celestijnenlaan 200D, 3001 Leuven, Belgium \\ email: nick@ster.kuleuven. be \\ ${ }^{2}$ Department of Physics \& Astronomy, University of Western Ontario, London, Canada \\ ${ }^{3}$ Astronomical Institute Anton Pannekoek, Universiteit van Amsterdam, Amsterdam, The \\ Netherlands \\ ${ }^{4}$ ESA/RSSD-ESTEC, Noordwijk, The Netherlands \\ ${ }^{5}$ George Washington University, Washington DC, USA \\ ${ }^{6}$ Leiden Observatory, University of Leiden, Leiden, The Netherlands \\ ${ }^{7}$ NASA-Ames Research Center, Space Science \& Astrobiology Division, California, USA \\ ${ }^{8}$ SETI Institute, 189 Bernardo Ave, Suite 100, Mountain View, CA 94043, USA
}

\begin{abstract}
We present the first results of an exploratory VLT/X-Shooter survey of near-infrared diffuse interstellar bands (DIBs) in diffuse to translucent interstellar clouds. These observations confirm the presence of recently discoved NIR DIBs and provide more accurate rest wavelengths and line widths. Example spectra are shown for the reddened, $A_{V} \sim 10$ mag, line-of-sight towards the distant binary system $4 \mathrm{U} 1907+09$.
\end{abstract}

Keywords. infrared: ISM, ISM: lines and bands, ISM, clouds

\section{Introduction}

The optical wavelength range has been extensively explored in terms of diffuse interstellar bands (Herbig 1995; Sonnentrucker, this volume). However, a few unsuccessul attempts have been made to find diffuse interstellar bands in the ultra-violet (see e.g. Bhatt et al., this volume). Investigations of the near-infrared wavelength range have also been limited, but were at least somewhat succesful in nature.

Joblin et al. (1990) detected in their moderate resolution $(R \sim 10000)$ J-band (1.1$1.35 \mu \mathrm{m})$ observations two near-infrared diffuse bands, at 11797 and $13175 \AA$, that correlated well with reddening. Adamson et al. (1994) searched for the $13175 \AA$ bands in dense environments but could only report upper limits. Subsequently, Foing \& Ehrenfreund (1994) discovered two additional near-infrared bands at 9577 and $9632 \AA$ which they assigned to two $\mathrm{C}_{60}^{+}$bands expected in this range from laboratory measurements.

Within the context of the PAH-DIB hypothesis (see e.g. Cox 2011 for a recent summary of this concept proposed in the mid-1980s) the presence of numerous electronic transitions in the near-infrared astrophysical spectra is expected for large ionised PAHs. Despite earlier predictions, it was only in 2011, that Geballe et al. recognized 15 new diffuse interstellar bands in the H-band $(1.5-1.8 \mu \mathrm{m})$, but none in the K-band $(2.0-2.4 \mu \mathrm{m})$, using the low-resolution $(R \sim 4000)$ NIFS instrument on Gemini North (see also Geballe, this volume). 

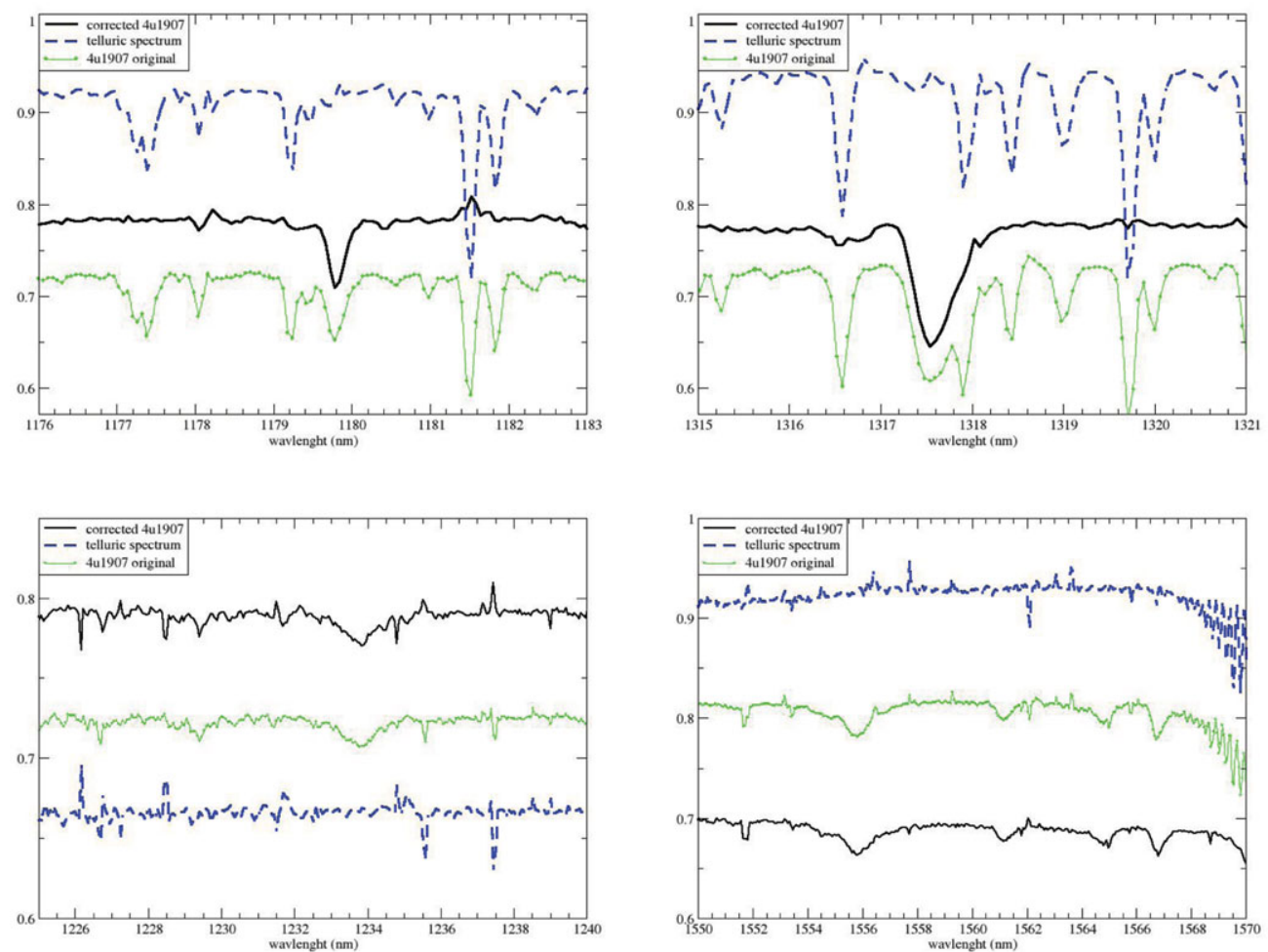

Figure 1. Near-infrared DIBs in the reddened, $A_{V} \sim 10 \mathrm{mag}$, line-of-sight towards the distant, 5 kpc, high-mass x-ray binary, 4U 1907 (Cox et al. 2005). Top left: $11797 \AA$ AIB; top right: $13175 \AA$ DIB, bottom left: $12330 \AA$ candidate DIB; bottom right: 15557, 15610, 15645, and $15665 \AA$ DIBs.

\section{VLT/X-Shooter near-infrared DIB survey}

In the past years we have obtained moderate resolution $(R \sim 16000)$ X-Shooter spectra $[0.3$ to $2.4 \mu \mathrm{m}]$ of a small set of well-known diffuse cloud lines-of-sight that probe simultaneously the DIBs in the optical and near-infrared. Here we present preliminary results from this survey, focusing on the reddened line-of-sight towards $4 \mathrm{U} 1907$. Full details and discussion of the results will be given in a forthcoming publication (Cox et al. 2014). There we will also provide relations between NIR DIBs, extinction and optical DIBs. Furthermore, accurate rest wavelengths (air) were derived by correcting for the radial velocity of the interstellar component. In addition we propose several new NIR DIBs between 1.0 and $1.3 \mu \mathrm{m}$, while we also find no apparent interstellar features beyond $1.8 \mu \mathrm{m}$.

The X-Shooter spectra confirm that the newly identified near-infrared DIBs are interstellar in nature. Several of the selected targets are relatively nearby and probe single cloud environments. Hence, we also derive accurate rest wavelengths and (smaller) values for the intrinsic widths of the near-infrared DIBs. From the observed integrated equivalent width of these new near-infrared DIBs we constrain the product of their relative abundance and oscillator strength. The total equivalent width, normalised to reddening, of the dozen NIR DIBs is $\sim 1.5 \AA$ (compared to total absorption strenght of $\sim 30 \AA / \mathrm{E}(\mathrm{B}-\mathrm{V})$ ) similar to the strength per unit reddening of the dozen strongest optical DIBs.

In Figure 1 we show several NIR DIBs in the spectrum of 4U 1907. The top trace refers to the tellurc standard spectrum, the lower trace the observed science spectrum and the 


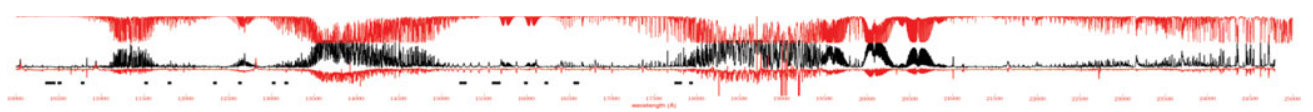

Figure 2. Observed near-infrared [1.0 to $2.45 \mu \mathrm{m}$ ] X-Shooter spectrum of HD 183143 (middle black trace). A simulated atmospheric transmission spectrum is also shown (top red trace) together with the telluric corrected spectrum (bottom red trace). Black squares indicate positions of known near-infrared diffuse bands.

middle trace to the telluric corrected spectrum. We note that with a proper choice of the standard star the telluric correction is very efficient leaving only minor residuals in the regions of interest for the current set of known NIR DIBs. The caveat is of course that there are several ranges, in the 'gaps' between the adjacent J, H, and K bands, where the atmospheric transmission is very low or even zero. Here correction with standards does not yield any satisfactory results. This is illustrated in Fig. 2 which shows the observed and telluric corrected DIB spectrum of HD 183143 (aka "Herbig's star") as well as that of the atmospheric transmission. To recognize DIBs in these wavelength ranges it will be necessary to avoid the atmosphere alltogether and utilize a infrared space telescope such as the JWST.

\section{Conclusion and future work}

The recent leaps forward in terms of NIR DIBs is very promising (see both contributions by Geballe and Zasowski in this volume), however, it also reveals that fundamentally there appear to be far fewer interstellar bands between 1.0 and $1.8 \mu \mathrm{m}$ compared to the narrower optical range $(0.5-1.0 \mu \mathrm{m})$. Also the lack of DIBs beyond $1.8 \mu \mathrm{m}$ might in itself contain information on the whole set of DIB carriers. In this respect it is paramount that we should make an effort to understand in detail how the NIR DIBs are connected to the optical DIBs as well as to other (atomic \& molecular) ISM constituents. Follow-up of NIR DIBs at higher spectral resolving powers may give additional information on their carriers, in particular if substructure or profile variations can be detected.

\section{Acknowledgements}

We thank the members of the local organising committee for their tireless efforts that helped make this symposium a success.

\section{References}

Cox, N. L. J. 2011, EAS Publication Series, 46, 349

Cox, N. L. J., Kaper, L., Foing, B. H., \& Ehrenfreund, P. 2005, A\&A, 438,187

Cox, N. L. J., Cami, J., Kaper, L., Ehrrenfreund, P., Salama, F., Ochsendorf, B. B., Van Hooff, S., \& Foing, B. H., $A \& A$, in preparation.

Foing, B. H. \& Ehrenfreund P. 1994, Nature, 369, 296.

Geballe, T. et al. 2011, Nature, 479, 200

Herbig, G. 1995, ARAESA, 33, 19

Joblin, C. et al. 1990, Nature, 346, 729 